\title{
Stars of the southern hemisphere proposed for the Hipparcos mission
}

\author{
J.M. Rousseau, J.P. Périé and M.T. Gachard \\ Observatoire de Bordeaux, INSU/CNRS-Université Bordeaux 1, B.P. 89, F-33270 Floirac, France
}

Received May 29; accepted August 29, 1995

\begin{abstract}
As part of the preparation for the Hipparcos mission, we determined the positions of 96800 already listed stars and 21265 previously unlisted companions, using information gathered by the ESO Quick Blue Survey. B1950 and J2000 system coordinates are given for each star. The magnitudes and spectral types are given in addition to the exact date of the observation (approximately 1976). PPM, HIC, SAO, HD, CoD, CPD, CPC, ADS, IDS, BD, LHS and $\mathrm{V}^{*}$ identifiers are given systematically whenever they exist as well as two supplementary identifiers selected from 43 other catalogues. Comments where necessary to complete the information. This catalogue is available from the Centre for Astronomic Data at Strasbourg.
\end{abstract}

Key words: astrometry — catalogues

\section{Presentation of the catalogue}

\subsection{Origin of the catalogue and observations}

In 1983, we were asked to participate in the compilation of the INPUT CATALOGUE for the HIPPARCOS mission.

This catalogue includes all the objects observed by the satellite (Turon et al. 1992). One of the conditions required for the correct running of this project was knowing, in advance, the position of each object in the programme with an accuracy of $1.5^{\prime \prime}$ for each coordinate; $0.5^{\prime \prime}$ being unofficially desired. Although this requirement was satisfied for stars included in more recent astrometric catalogues, (FK5, AGK3 and PERTH 70), it was not the case for those described exclusively in older catalogues or in personal lists of certain proposers. The only reliable solution, for a certain number of cases, was to redetermine the positions, in a uniform manner, based on recent photographic observations or new meridian observations. The complete programme of compilation and observation carried out for this project was described by Jahreiß et al. (1992).

We have presented below all the measurements taken at Bordeaux, from photographic plates, for stars in the southern hemisphere. This was, in fact, the region of the sky in which the greatest number of problems and the least accuracy was encountered, since the high density

Send offprint requests to: J.M. Rousseau catalogues were either old ( $\mathrm{CoD}, \mathrm{CPD})$ or approximate (HD).

It soon became apparent that we would have to use copies of the ESO Quick Blue Survey available from the Paris-Meudon Observatory. This recent collection is made up of 606 plates, in $30 \times 30 \mathrm{~cm}$ format, i.e. 29 square degrees, with a scale of $0.0148 \mathrm{~mm}$ per arcsecond and an exposure time of 1 hour. The magnitude limit was approximately 20, well above our requirements. All these characteristics have been published (West \& Schuster 1982).

However, the coverage of this collection is only for declinations from -17.5 to -90 degrees. We were thus limited to observations within this zone, equivalent to approximately one third of the sky. The reference catalogue used was Perth 70, which offers very recent observations, and which was the only reasonably dense astrometric list (approximately 1 star per square degree) covering the zone in question in 1985 (Høg \& von der Heide 1976).

We checked that the use of glass copies instead of the original plates did not affect the accuracy. Comparative measurements performed at Garching (where the originals are stored) using the Optronics measuring instrument, showed that the results would not be degraded. Indeed, for star-rich plates, the contrast quality is better on the copies, thus improving the ease and precision of sightings. 


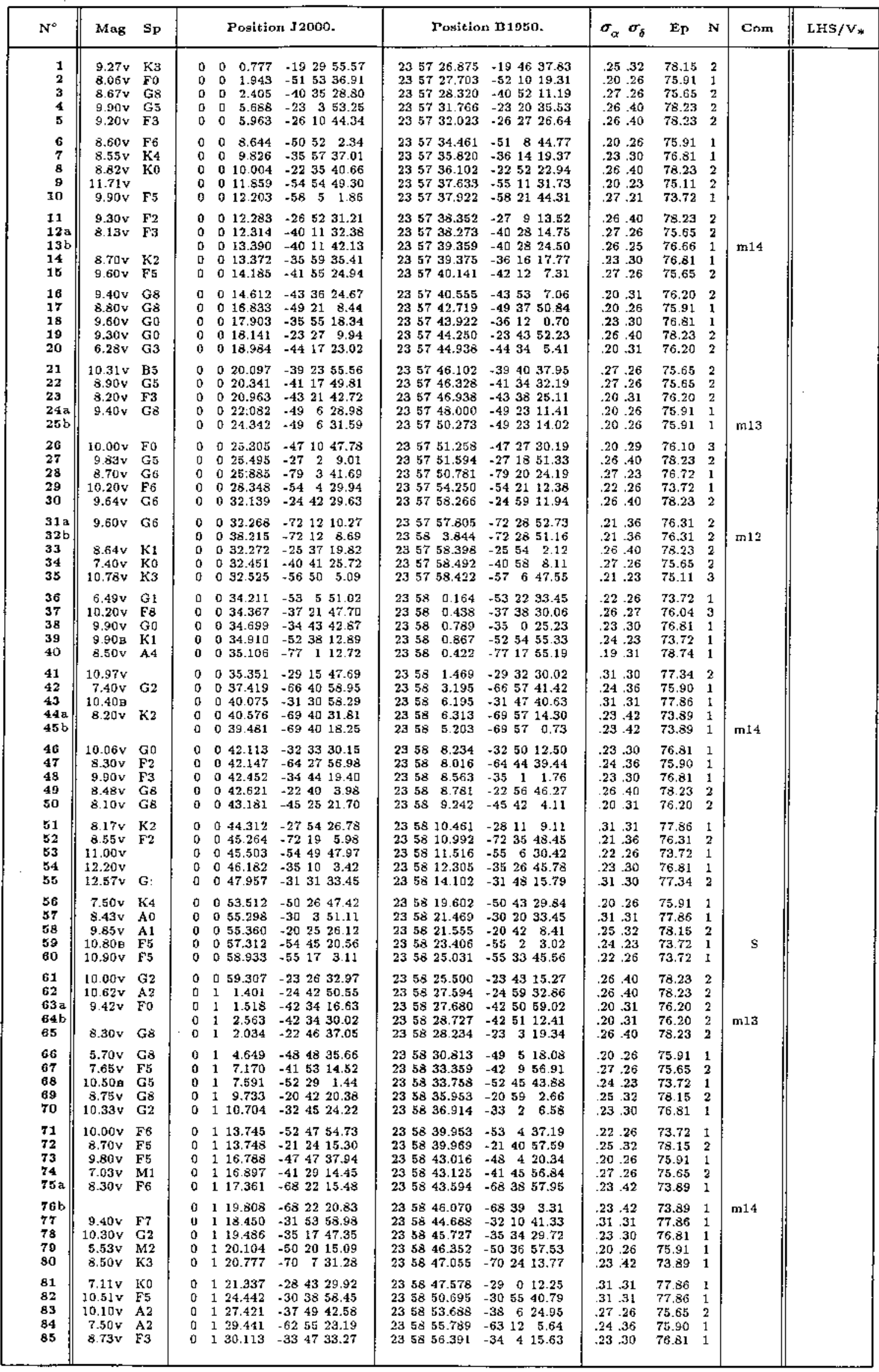




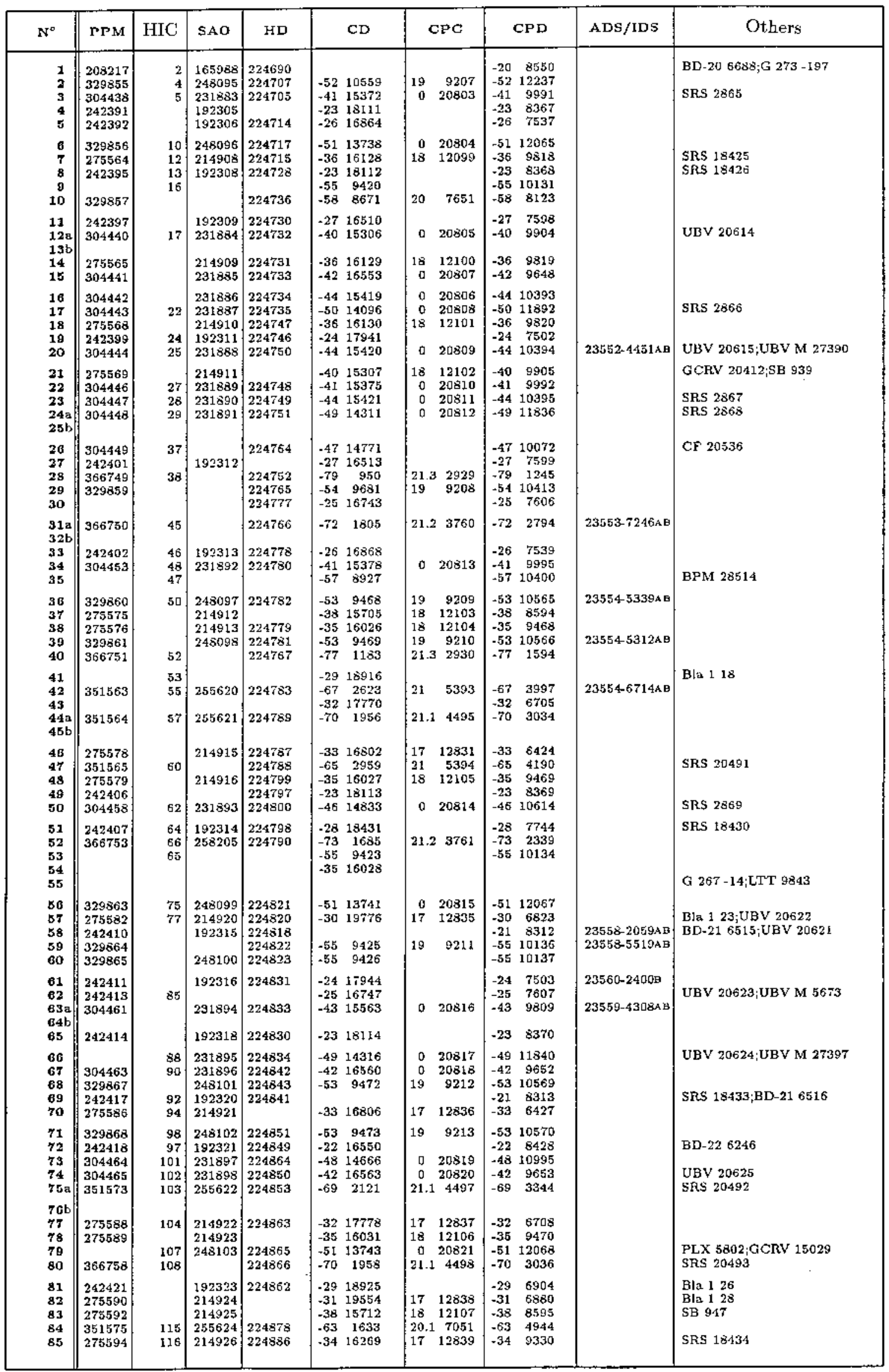




\subsection{Catalogue composition and choice of objects}

Based on the proposals for the Hipparcos observation programme, the INCA CONSORTIUM team provided us with a list of 84323 objects, with no repetitions; many more than the 46785 stars of the Hipparcos Input Catalogue in our zone of 14225 square degrees. We decided to treat all the proposed stars equally. This list was very close to the total number of proposals for the zone under consideration.

After measuring, and for various reasons, 389 objects were missing, including:

- 72 variable stars with incorrect coordinates.

- 13 stars ignored since they were too bright to be measured.

- 40 anonymous stars with insufficient data.

- 17 high speed and faint stars, whose initial coordinates were inaccurate (L, LP, BPM, etc.).

- 150 companions very close to a bright main object and obscured by its halo.

- 41 stars whose identification was too uncertain.

To this initial list we added:

- Unexpected companions of stars included in the programme, that might induce errors when the star's position and magnitude was measured by the satellite (as a function of their distance from the main object and their brightness). We determined their position with the same accuracy as for the main objects and estimated the magnitude of most of them. The visible companions in question are less than approximately $30^{\prime \prime}$ from the main object and have an estimated magnitude of up to 15 . Systems detected in this way do not appear in the ADS and IDS catalogues. No indication of a dynamic link exists. Only the apparent proximity is indicated.

- Objects selected in regions not covered by any of the proposals that constitute the programme. When regions having a low stellar density were detected in this list, we looked for identifiable objects and added them to the catalogue.

- Companions not requested, but already known and included in the ADS and IDS catalogues.

\subsection{Measurements}

The plates were measured using one of the observatory's Zeiss comparators. The instrument had been slightly modified before the start of the work by mounting Heidenhain rules in order to automate the reading of the $X$ and $Y$ coordinates and to eliminate human error. Furthermore, this device, which is commonly used, enabled readings to be directly transmitted to the computer, thus avoiding transferral error.
The construction of the rules enables a display to be obtained to the nearest $0.1 \mu$, however the average measurement scatter is $2 \mu$. As a result of the relatively long exposure time for these plates, bright stars are shown as large images and are affected by diffraction, since the strips supporting the plate holder generate a parasitic image of 4 to 8 branches. Up to approximately mag 10, the only measurement possible is to sight this cross. This was the case for all the reference stars included in the Perth 70 catalogue. Whilst this procedure is not without flaws, the diameter of the images prohibits any other solution. In order for this work to have been carried out with greater precision, for a programme such as this which includes stars with magnitudes from 2 to 14, it would have been necessary to have several plates for each field with increasing exposure times; which was not the case. Images fainter than $B=10$ were circular without spikes.

All the measurements for any one plate were carried out by one operator, as quickly as possible. Each reference star was measured 4 times, and objects twice (since the plate was observed first in its normal position, and then after the optical system was rotated through 90 degrees). Two operators performed all the measurements, over a period of 25 months.

\subsection{Reductions}

The equatorial coordinates for the centre of each plate are known approximately (West \& Schuster 1982).

On average, there were 32 reference stars per plate (varying from 20 to 43 depending on the region).

A file was created for each plate, which after measuring included:

- equatorial coordinates for each reference star, on the day of the observation.

- rectangular coordinates for the same stars.

- rectangular coordinates for the objects.

Using a traditional method (Barlier \& Muller 1964; Dodd 1972), this data enabled the determination of the value of the 10 coefficients (or plate constants) of the third order polynomials relating each equatorial coordinate to rectangular coordinates.

The analysis and evaluation of all the coefficients resulting from the reduction of the 606 plates was performed separately (Ducourant, Thesis 1989).

For each plate, the root-mean-square error is given by

$$
\sqrt{\frac{\Sigma(\mathrm{o}-\mathrm{c})^{2}}{(n-10)}}
$$

Any reference star whose (o-c) term was greater than 2.5 times the root-mean-square error of the plate, in right ascension or in declination, was rejected and the reduction performed again. 
For all the 18653 reference stars used, the root-meansquare deviation was: $0.25^{\prime \prime}$ in right ascension and $0.29^{\prime \prime}$ in declination

\subsection{Identifiers}

Particular attention was paid to finding all the principal identifiers for each object. The search covered 55 different catalogues in addition to maps and lists of galactic open clusters. The procedure followed was:

- Consultation of the Hipparcos Input Catalogue

- Consultation at the Centre for Astronomic Data in Strasbourg (CDS)

- Direct search in original works.

The identification of stars in galactic clusters is often difficult. For the Hipparcos mission a catalogue of positions of southern open cluster stars was independlently built, refered to catalogue SAO (Tucholke 1989). For 389 stars common to two catalogues, we have compared the identifiers. Without exception, the identifiers are similar.

584 stars did not have an identifier. These stars were faint objects, with magnitudes fainter than 10, proposed to the Hipparcos mission without being included in any catalogue.

\subsection{Comparison with the Carlsberg Automatic Meridian Catalogue}

The Carlsberg Automatic Meridian Catalogue, (Vols. 4-6, 1989-1992) was selected as a reference since it had been compiled using recent and impersonal meridian observations. Comparisons were not made for all the stars but by random local samplings, and for the zone from -17.5 degrees to -45 degrees which was common to the two catalogues. For each common star, the position was calculated, according to the CAMC at the ESO plate epoch (using the proper motion components given by this catalogue). The Bordeaux-CAMC differences for each coordinate were also calculated. The mean value, or systematic error is: $0.001 \mathrm{~s}$ in right ascension and $-0.055^{\prime \prime}$ in declination.

The standard deviations are: $0.22^{\prime \prime}$ in right ascension and $0.25^{\prime \prime}$ in declination.

It should be noted that the Bordeaux catalogue is referred to the Perth 70 catalogue (and thus to the FK4 system) whilst the Carlsberg Meridian Catalogue uses the FK5 system.

Isolated cases of differences of up to $0.5^{\prime \prime}$ were observed. They often correspond to stars located on the edges of the ESO plates, or to double stars that we measured separately in contrast to the Carlsberg Meridian who sighted the combination.

\section{Catalogue description}

The description follows the same order as the catalogue columns. Two sample pages of the catalogue are given at the back of this paper.

\subsection{Number $\left(N^{\circ}\right)$}

Numbers are given, from 1 to 118065, irrespective of the hierarchy of the object. The object's rank is recognised:

- for systems that are already known, by the suffix (A, $\mathrm{B}, \mathrm{C}$, etc.) to the ADS or IDS identifier.

- for previously unlisted systems, that we detected, by the letter "a" after the main object, and letters b, c, d, etc. after the companions.

The star entries follow the order of the object's right ascension (J2000), except for previously unlisted companions (with b, c, d, etc.) which immediately follow the associated main object. In other words, it is the right ascension of the main object "a" which places the system in the catalogue. This should avoid annoying "mix-ups" with other objects or other systems.

In accordance with the Centre for Astronomic Data at Strasbourg, we suggest to use the acronym SHB (Southern Hemisphere Bordeaux).

\subsection{Magnitude (Mag)}

It was not possible to add the measurement of photographic magnitude to our work. We have therefore drawn this data from various sources, hence their lack of homogeneity. For the same reasons, the magnitudes are either in $B$ or $V$. Our sources were:

- the HIC catalogue.

- the Centre for Astronomic Data at Strasbourg.

- original catalogues (HD, CoD, CPD).

- ADS and IDS catalogues for companions not included in the programme.

- the list of the sum of proposals.

- our personal estimations, made by rapid comparison with nearby stars.

This process may lead to errors, depending on the colour of the object, of up to 1.5 magnitudes. It was used in cases where data was not present in other catalogues. The values given should only be used as an indication.

\subsection{Spectral type $(S p)$}

The same reservations apply, to an even greater degree, to the indications of the spectral type. Since this parameter is only given by way of an indication, we have kept only 2 characters corresponding to the principal types. 


\subsection{J2000 and B1950 positions}

We have continued with the B1950 positions, which are still widely used today. They refer to the FK4 system, as does the Perth 70 catalogue which provided our reference stars.

We just precessed the B1950 coordinates originally measured to 2000 (Lieske et al. 1977 and 1979). We did not apply the regional systematic differences between the FK5 and FK4 system given e.g., with the FK5.

2.5. Precision, epoch and number of observations $\left(\sigma_{\alpha}, \sigma_{\delta}, \mathrm{Ep}, \mathrm{N}\right)$

- $\sigma_{\alpha}, \sigma_{\delta}$ : In principle, each star should only have been examined once. However, the plates from the ESO Quick Blue Survey overlap, to a greater or lesser extent. Stars located on the edges may therefore have been measured 2 or even 4 times (in a corner). Furthermore, certain plates were measured several times. Occasionally both cases occurred for the same plate. The following solutions have been adopted:

- Several positions of an object for the same plate: The mean position of the object is used, on the plate epoch.

- One or more positions of an object on more than one plate. We give:

the average of the positions

the average of the plate epochs

the resulting deviation for each coordinate:

$$
\sqrt{\frac{\Sigma(\sigma)^{2}}{n}}
$$

where $\sigma$ is the deviation resulting from each reduction, given in arcsecond, as has already been defined, for reference stars only.

- Ep: The relatively small time period separating two neighbouring plates allowed us to use mean positions (the plates were exposed between 30/03/1973 and $22 / 02 / 1979)$. In many cases, neighbouring plates correspond to very similar epochs.

The epoch of each plate is known to the nearest second (West \& Schuster 1982). This precision is not required for this type of catalogue. We therefore only give the epoch (Ep) to the nearest 0.01 year.

- N: Indicates the number of measurements carried out for this star.

\subsection{Comment (Com)}

This column contains a wide range of information: $m$.. $\quad$ The estimated magnitude of previously unlisted companions, to the nearest $1 \mathrm{mag}$, given by way of an indication only (varying from 10 to 16).

2 to 39 Indicates that the star forms part of a group of $n$ stars, in which each star is less than $50^{\prime \prime}$ from another. The most frequent numbers are 2 and 3 and are often observed in relation to systems cited by the ADS and IDS catalogues. The other numbers relate in general to members of galactic open clusters. This number should be primarily considered as a signal indicating the proximity of other objects and thus the risk of confusion or identification error.

$a=b \quad$ Components $a$ and $b$ appear to be equal in magnitude.

D The star in question may be a double star (69 cases).

S The star in question is part of a pair that is too close together to enable the measurement of the two components.

? A doubt exists as to the identification of the components. The allocation of the letters a and $\mathrm{b}$ is arbitrary.

* See notes.

\section{7. $L H S / V^{*}$}

Gives both the stars included in the Luyten Half-Second Catalogue (Luyten 1979) (stars with proper motion larger than $0.5^{\prime \prime}$ annually) and variable stars taken from the General Catalogue of Variable Stars (GCVS, Kholopov 1990). These categories may be combined in the same field since no star is included in both catalogues. Variable stars designated uniquely by a Greek letter and the constellation are mentioned in the notes.

2.8. PPM, HIC, SAO

Give the numbers taken from the following catalogues:

- Positions and Proper Motions (Bastian et al. 1992).

- HIC (Turon et al. 1992).

- Smithsonian Astrophysical Observatory Star Catalogue (1966).

2.9. $H D$

Gives numbers taken from the:

- Henry Draper Catalogue (Cannon \& Pickering 19181949).

- Henry Draper Extensions (Cannon 1925-1936; Cannon \& Mayall 1949). 
When a star belonging to the HD catalogue is double or multiple (according to ADS or IDS) the letter A is never placed after the HD number of the main object. However, the companions are assigned the same HD number followed by the same suffix B, C, etc. as in the ADS or IDS identifier.

The character + following an HD identifier indicates that the entry relates to two neighbouring stars in our catalogue (one or the other or the sum of both) for which the doubt has not been resolved (inaccuracy of the positions in the HD catalogue and absence of comments). It does not form part of the identifier.

\subsection{0. $C D$ and $C P D$}

Respectively gives the numbers from the Cordoba Durchmusterung (Thome 1892, 1894, 1900, 1914 and Perrine 1932), and those from the Cape Photographic Durchmusterung (Gill 1903).

The HD Convention has not been taken into consideration. The two CD and CPD identifiers are given regardless of the declination of the star under consideration.

When a star belonging to one of these catalogues is double or multiple, according to ADS or IDS, the identifier is given for each component, accompanied by the same suffix A, B, C, etc. as the ADS or IDS identifier.

The character + following an CD or CPD identifier indicates that the entry relates to two neighbouring stars in our catalogue (one or the other or the sum of both) for which the doubt has not been resolved (inaccuracies in the $\mathrm{CD}$ or CPD catalogue and absence of comment). It does not form part of the identifier.

Certain CD or CPD identifiers are followed by a "p" or an "s". Should two stars be close together, correspond to a single CD or CPD identifier, and not be included in the ADS and IDS catalogues, this notation enables the components to be more clearly designated. This system has been adopted by the Centre for Astronomic Data in Strasbourg.

\subsection{1. $C P C$}

Gives the numbers from the Cape Photographic Catalogue for 1950.0 (Jackson \& Stoy 1954-1968).

In 4 cases, the character + following a CPC identifier indicates that this entry relates to two neighbouring stars in our catalogue. It does not form part of the identifier. This fusion is indicated in a note in the actual CPC catalogue.

Stars designated by CPC 0 nnnnn are included in the "Catalogue of the positions and proper motions of stars between declinations $-40^{\circ}$ and $-50^{\circ \prime \prime}$ (Hoffleit 1970).

\subsection{ADS/IDS}

Contains the identifiers from the New General Catalogue of Double Stars (Aitken 1932) and the Index of Double Stars (Jeffers et al. 1963).

It was possible to group the identifiers since their formats are very different. The presence of a 2-letter suffix $(\mathrm{AB}, \mathrm{BC}$, etc.) indicates that we were unable to separate the two components from fused or very close images.

\subsection{OTHERS}

The identifiers described in the previous 9 columns correspond to the major catalogues in common use. However it was necessary to mention others, in a less systematic way, depending on the space available. These are often local or smaller catalogues. Priority was given to the BD (Schönfeld 1886), L, LP, LTT (Luyten 1957, 1979, 1980) identifiers and the numbers designating the members of galactic open clusters. The presence in this column of BD identifiers is justified by the zone of less than 6 degrees included in the Bonner Durchmusterung.

We have occasionally given additional identifiers in the notes, indicated by a * in the Com. column.

\section{Conclusion}

This catalogue has not been compiled simply for astrometric purposes, but primarily to prepare for the HIPPARCOS satellite mission. Having fulfilled this role, it has been adapted to meet the requirements of observers and those who would like to identify objects with varied names, hence the care taken in terms of cross-referencing identifications from 55 catalogues. Numerous ambiguous cases have been individually dealt with, in particular in the galactic open clusters and multiple systems through referring to original articles and reference works. The catalogue's principal characteristic is its homogeneity, since it has been compiled from plates taken by one instrument, measured using one machine, in a short time, by just two operators, and reduced by one method, the results of which were submitted to identical criteria.

This catalogue has been filed at the Centre for Astronomic Data in Strasbourg and is also available in printed form.

Acknowledgements. This work was carried out following checks performed at Garching using the Optronics measuring instrument on the original plates. We would like to thank R.M. West for giving us permission to use these plates in these exceptional circumstances. The library of the Paris-Meudon Observatory successively lent us the 606 plates taken by the ESO Quick Blue Survey. Without these documents and the guarantee of their high quality, this work would not have been possible. The workshop at the Bordeaux Observatory carried out a great deal of 
essential work in modifying and maintaining our Zeiss instrument. The Computing Department designed the acquisition and measurement data transfer systems. The help of everyone involved is gratefully acknowledged.

Our particular thanks go to C. Turon, who, together with her team, advised us and provided documentation over several years. Without her, this catalogue would not have been compiled.

\section{References}

Aitken R.G., 1932, New General Catalogue of Double Stars within $120^{\circ}$ of the North Pole, Carnegie Inst. of Washington

Barlier F., Muller P., 1964, Bull. Astr. Obs. Paris XXIV, 29

Bastian U., et al., 1992, PPM South Star Catalogue, Astronomisches Rechen-Institut, Heidelberg

Cannon A.J., Pickering E.C., 1918-24, Henry Draper Catalogue, Ann. Astron, Obs. Harvard College, Vols. 91-99

Cannon A.J., 1925, Henry Draper Extension I, Ann. Astron, Obs. Harvard College, Vol. 100

Cannon A.J., Walton Mayall M., 1949, Henry Draper Extension II, Ann. Astron, Obs. Harvard College, Vol. 112

Carlsberg Meridian Catalogue La Palma, Vol. 4 (1989), Vol. 5 (1991), Vol. 6 (1992)

Dodd R.J., 1972, AJ 77, 306

Ducourant C., 1989, Thèse de Doctorat Bordeaux

Gill D., Kapteyn J.C., 1896-1897-1900, The Cape Photographic Durchmusterung, Ann. of the Cape Observatory, Vol. III-V

Hoffleit D., 1970, Catalogue of the positions and proper motions of stars between declinaisons $-40^{\circ}$ and $-50^{\circ}$, Transactions of the Astronomical Observatory of Yale University,
New Haven, Vol. 30

Høg E., von der Heide J., 1976, Abhandlungen aus der Hamburger Sternwarte, No. 9

Jackson J., Stoy R.H. 1954-1968, Cape Photographic Catalogue for 1950.0., Ann. of the Cape Observatory, Vols. XVIIXXII

Jahreiß H., et al., 1992, A\&A 258, 82

Jeffers H.M., van den Bos W.H., Greeby F.M., 1963, Index Catalogue of Visual Double Stars, Univ. California, Publ. Lick Obs., Vol. XXI

Kholopov P.N., et al., 1990, General Catalogue of Variable Stars, Fourth Edition, Moscow, Nauka Publ. House

Lieske J.H., et al., 1977, A\&A 58, 1

Lieske J.H., 1979, A\&A 73, 282

Luyten W.J., 1957, Luyten Two-Tenths Catalogue, Univ. Minesota, Mineapolis

Luyten W.J., 1979, Luyten Half-Second Catalogue, Univ. Minesota, Mineapolis

Luyten W.J., 1980, New Luyten Two-Tenths Catalogue, Univ. Minesota, Mineapolis

Perrine C.D., 1932, Cordoba Durchmusterung, Results of National Argentine Observatory, Vol. 21

Schönfeld E., 1886, Bonner Sternverzeichniss, Vierte section, A. Marcus, Bonn

Smithsonian Institution, 1966, Smithsonian Astrophysical Observatory Star Catalogue, Washington D.C.

Thome J.M., 1892-1914, Cordoba Durchmusterung, Results of National Argentine Observatory, Vols. 16-21

Tucholke H.J., 1989, A\&AS 78, 187

Turon C., et al., 1992, The Hipparcos Input Catalogue, ESA SP-1136, Vols. 1-7

West R.M., Schuster H.E., 1982, A\&AS 49, 577 